\section{Claves para el conocimiento de la configuración espacial de una torre banderiza: la Torre de Murga (siglos XIV-xv)}

ISMAEL GARCía GÓMEZ

Unidad Asociada CSIC/Universidad del País Vasco. Grupo de Arqueología Tardoantigua y Medieval. Arqueología de la Arquitectura (GATMAA)

\section{Resumen}

En este trabajo se presentan esquemáticamente los resultados del análisis arqueológico de la torre banderiza de Murga (Ayala, Álava), y se hace uso de ellos -en combinación con los resultados arrojados por otras torres similares- para establecer una propuesta de interpretación de la articulación espacial de este tipo de edificaciones. Entre otras cosas se resalta la importancia del cadalso y la inexistencia de un acceso por la planta baja. Finalmente se reseñan los criterios que, a juicio de los autores, deben ser seguidos a la hora de analizar una torre de estas características.

Palabras clave: Torres banderizas; análisis estratigráfico; análisis espacial; Murga.

\begin{abstract}
This work schematically presents the results of archaeological analysis of the torre banderiza (a type of tower particular to the Basque Country) of Murga (Ayala, Álava) and makes use of these - in combination with results found at similar towers - to establish a proposal of interpretation of the spatial basis of this type of tower. The work highlights, among other aspects, the importance of the platform/ scaffold and the inexistence of ground floor access in this type of construction. Lastly the work summarises the criteria which, in the authors' opinion, should be followed in analysing a tower of these characteristics.
\end{abstract}

Key Words: Torres banderizas; stratigraphic analysis; spatial analysis; Murga
Empezar de cero. Bien podría resumir esta frase la impresión del arqueólogo que se enfrenta al estudio de un edificio como éste de la torre de Murga, aunque se trate de una sensación que no corresponda con la realidad. El utillaje conceptual propio de la Arqueología de la Arquitectura y su herramienta básica, el análisis estratigráfico, han contribuido de manera tan determinante a la comprensión del hecho constructivo que, en muchos casos, el arqueólogo se ha visto en la necesidad de replantear cuestiones aparentemente ya zanjadas por la historiografía.

El de las torres banderizas es un fenómeno sobre el que se ha escrito mucho, pero que permanece esencialmente en la esfera de lo desconocido. Numerosas veces ha sido abordada la descripción de los elementos arquitectónicos que las componían, el uso hipotético de cada una de las plantas de su interior o la narración genealógica de los linajes que las habitaron, y sin embargo, en raras ocasiones la investigación se ha preocupado por conocer los antecedentes de la tradición constructiva que les dio origen o por saber del impacto social de su edificación.

El trabajo que aquí presentamos, fruto de un estudio realizado en el año 2001 en la torre de Murga (Ayala, Álava), pretende trascender lo puntual del caso y plantear una reflexión genérica sobre este tipo de edificios. Por un lado, esta reflexión se articulará en torno a las claves metodológicas sobre las que debería incidir, a nuestro juicio, el análisis de una torre, y por otro, en torno a las que consideramos como claves para la interpretación funcional de su espacio.

No partimos de cero. Esa es la realidad, pero debemos deshacernos de todas las preconcepciones de origen dudoso que abundan en la caracterización de la vida del pariente mayor y por extensión, de su vivienda. Para alcanzar, a largo plazo, la comprensión del modelo banderizo de apropiación y control del territorio, se hace necesario partir, en un proceso fundamentalmente deductivo, de cuestiones básicas acerca de la utilización del espacio.

\section{LA CASA-TORRE, CONSECUENCIA INEVITABLE DE UNA FORMA DE ENTENDER EL TERRITORIO}

Los señores «de la guerra y de la tierra» vieron en el paisaje una fuente de recursos económicos sobre los cuales extender su dominio y, en la casa-torre, la forma idónea de articular el control de ese territorio en beneficio propio. La construcción de uno de estos edificios era, ante todo, la expresión de una voluntad de dominio formulada en clave espacial. Así, a mediados del siglo XIV, los Murga pretendieron hacer indiscutible su poder sobre la zona Meridional del valle de Ayala (Fig. 1). 


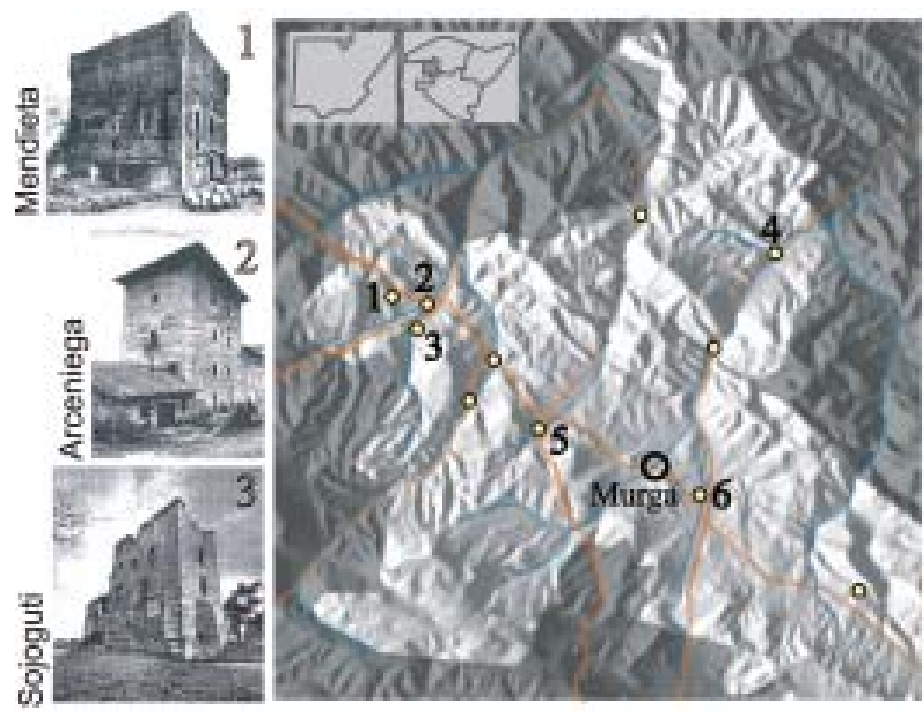

Este dispositivo de poder señorial estaba diseñado principalmente para atender a necesidades militares. La estructura del recinto y su distribución espacial estaban pensadas para poder llevar a cabo una resistencia efectiva ante el enemigo y sólo en segundo lugar, para atender a las necesidades domésticas, reservándose un hueco considerable a la vivienda del señor.

Aquellos que vivían en su interior no tenían otra opción que la de aceptar unas condiciones de habitabilidad impuestas, lo cual incidía, no sólo en la funcionalidad que adquiría cada uno de los espacios del interior del inmueble, sino sobre todo, en la forma en que se producía el juego de las relaciones sociales. La torre fue el producto de una sociedad bajomedieval en crisis ${ }^{1}$, y, al mismo tiempo, uno de los factores clave en el modelado y perpetuación de su estructura.

\section{LA NECESIDAD DEL ANÁLISIS ESTRATIGRÁFICO}

Hasta hace pocos años no existían las herramientas teóricas y metodológicas que permitieran comprender la secuencia de hechos constructivos que componen un edificio, es decir, la articulación diacrónica de sus fábricas. Por ello era hasta cierto punto comprensible que los análisis de las

\footnotetext{
${ }^{1}$ Es fundamental partir de la comprensión de la lucha de bandos no sólo como un conflicto horizontal internobiliario sino como, en palabras de Díaz de Durana, "un conjunto de conflictos sociales, con distintos niveles de expresión (...) relacionados entre sí por un denominador común: la crisis del sistema feudal», (Díaz DE Durana, 1998: 40). Dado que la extensión de este artículo no permite sintetizar adecuadamente el interesante debate historiográfico en torno a la llamada lucha de bandos, pilar fundamental de nuestra investigación, invitamos a la lectura, aparte de la citada obra de 1998, de: LeMA PueYo J.A. et al., 2000, Los señores de la guerra y de la tierra: nuevos textos para el estudio de los Parientes Mayores guipuzcoanos (1265-1548), San Sebastián.
}

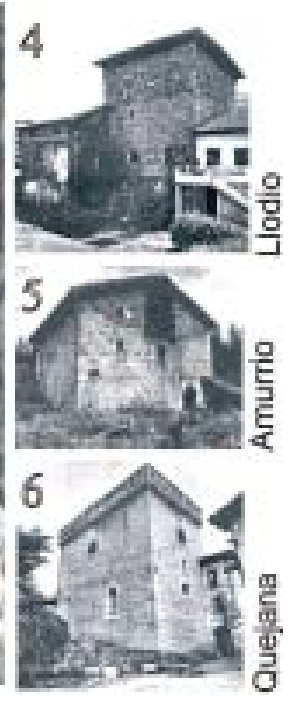

Fig. 1. Ubicación geográfica del valle de Ayala y representación esquemática del mismo. Sobre el mapa, en color anaranjado, las principales rutas comerciales, y en azul, los ríos. Como se puede comprobar, la torre de Murga y sus semejantes se situaban preferentemente en las intersecciones de estas dos redes. (Fotografías: Agustín Peña. Publicadas en Portilla, 1972) construcciones se basaran en criterios analógicos y descriptivos. Pero en los últimos tiempos, hemos ido adquiriendo una concepción más amplia de la serie de hechos que constituyen el resultado final de las construcciones históricas, tomando conciencia de la heterogeneidad que es intrínseca al paso del tiempo sobre ellas. Y gracias a esta conciencia, nos hemos dotado de unas herramientas que permiten descodificar esa secuencia, segregando las diferentes articulaciones sincrónicas por las que ha pasado el conjunto objeto de análisis, pudiendo llegar a una comprensión analítica de su historia.

Pero, a pesar de que ya contamos con esta posibilidad, siguen siendo raras las ocasiones en las que, al estudio particular de un edificio, antecede un análisis arqueológico de las fábricas. Lo que debería ser una obligación (no sólo legal, en el caso de las restauraciones, sino también autoimpuesta por los investigadores), se salda las más de las veces con vagas apreciaciones sobre aquellos elementos arquitectónicos u obras cuya disparidad con respecto a la fábrica principal resulta evidente. Esto, unido a que el argumento estilístico, puramente formal, es admitido con pocas reticencias a la hora de establecer una cronología, ha permitido la confección y aceptación de modelos hipotéticos de torre que, a nuestro juicio, plantean serias dudas interpretativas.

Ante esta situación, y sin obviar el recurso a la tipología de las formas arquitectónicas y otros tipos de análisis, se hace necesario subrayar que el análisis estratigráfico es la conditio sine qua non para el establecimiento de una cronología válida en cualquier estudio histórico y constructivo de una edificación, puesto que sólo él permite saber qué elementos arquitectónicos han funcionado coetáneamente y cuales de ellos se han sucedido en el tiempo. 

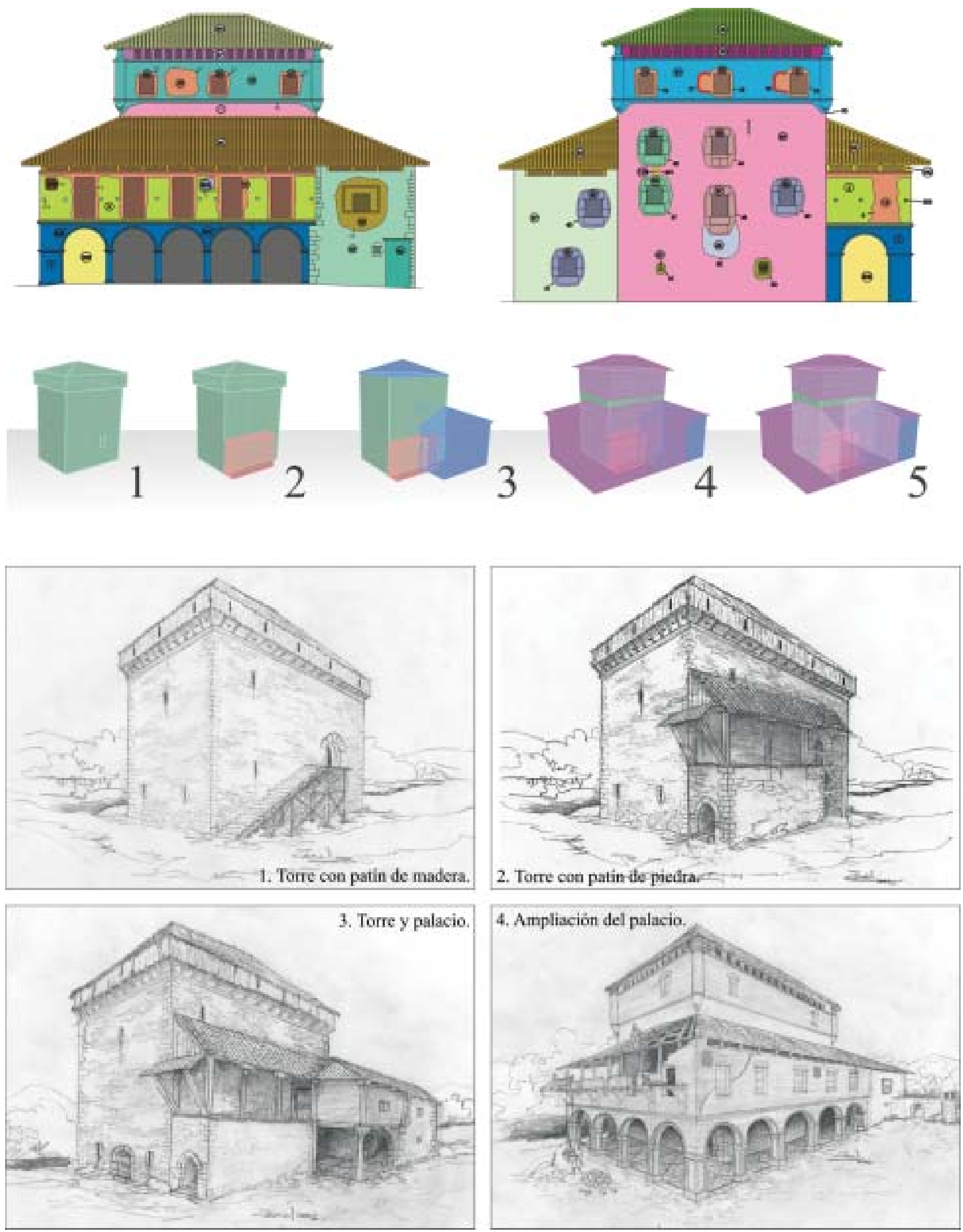

Fig. 2. En la zona superior. Dos de los croquis del análisis estratigráfico (a la izquierda la fachada $\mathrm{S}$ y a la derecha la N), los números corresponden a las unidades estratigráficas identificadas.

En la franja central y abajo. Fases de la evolución constructiva del edificio; la fase 5 corresponde a mediados del siglo xVIII, momento en el cual, el palacio es arrendado y se subdivide entre tres familias que lo utilizan como caserío. (llustraciones: Ismael García) 
En Murga, la aplicación del método estratigráfico ha puesto de relieve interesantes aspectos de la evolución del hábitat señorial que nos permitirán profundizar en el conocimiento del proceso de yuxtaposición de las estructuras sociales y familiares desde un punto de vista espacial, en su recorrido de los siglos XIV al XVI. En esta ocasión, sin embargo, nos concentraremos exclusivamente en algunas consideraciones acerca de la torre banderiza, dejando para futuras ocasiones el estudio del palacio renacentista que se adosó a ella con posterioridad (Fig. 2.).

\section{DESCRIPCIÓN RECONSTRUCTIVA DE LA TORRE DE MURGA (SIGLOS XIV Y XV)}

Físicamente, la torre de Murga era un recio edificio de planta rectangular $(9,50 \quad 13,15 \mathrm{~m}$.) que alcanzaba más de $11,8 \mathrm{~m}$. de altura. El aparejo de su fábrica era, y es, de mampostería caliza donde se alterna el material extraído de cantera por capas naturales con el material semielaborado (bozze), siguiendo hiladas más o menos regulares, aunque sinuosas. Los esquinales y recercos de vanos están realizados en sillares calizos de grandes dimensiones. La argamasa que cohesiona toda la obra es muy dura, abundante en cal y áridos. El grosor de sus muros en la base oscila en torno a los $1,35 \mathrm{~m}, \mathrm{y}$ todo ello se asienta sobre el nivel de roca natural, sin mediar solución de continuidad que funcione como zanja de cimentación.

El interior de la torre estaba dividido en tres pisos, comunicados por medio de escaleras. En la planta baja, presentaba cuatro pequeñas saeteras (enfrentadas dos a dos en los muros $\mathrm{E}$ y O); en la primera planta, según se ha podido constatar, sólo una saetera (como único vano además de la puerta de acceso) ubicada en el cierre norte, junto a la esquina $\mathrm{NO}^{2}$.La segunda planta, contaba con cuatro vanos, rematados en arco de medio punto; los de este nivel están centrados en cada uno de los paños, y a pesar de ser algo mayores que los de la planta baja, siguen siendo de reducidas dimensiones. La tercera planta correspondía al cadalso, que destacando sobre la línea de desplome de los muros de la torre, coronaba el edificio en todo su perímetro (Fig. 3).

No existía ningún acceso directo por la planta baja; la única entrada de la torre se encontraba en alto, en la primera planta del paño $S$. En un primer momento, a este ingreso

\footnotetext{
2 Creemos bastante probable la existencia de algún vano más, oculto tras los enlucidos que enmascaran las paredes de esta planta, tanto al interior como al exterior.

3 Entre las investigaciones realizadas por Grupo de Investigación en Arqueología de la Arquitectura de la UPV/EHU se incluyen los de otras torres como Martiartu y Madariaga en Vizcaya o Mártioda y Fontetxa en Álava.

4 Otra reciente aportación la encontramos en PALACIOS, 2001: 163-189.
}

se llegaba ascendiendo por una escalera de madera construida pegada a la fábrica, aunque poco después ésta fue sustituida por otra de piedra y rodeada de un muro defensivo (Fig. 2, fase 2).

El entramado de madera que divide el interior de la torre en la actualidad no es el que corresponde a la fábrica original. Aunque en sus rasgos fundamentales coincide con aquél, el análisis detenido de las ménsulas y mechinales que han quedado fuera de uso ha permitido poner de manifiesto importantes diferencias en la distribución y comunicación de los espacios, vitales para interpretar su funcionalidad (Fig. 3, croquis 1).

La simbiosis entre la estructura lígnea y el trabajo de albañilería de los paños perimetrales que se desprende de la articulación entre aparejo, huecos y apoyos, es total. De la forma y configuración de los mechinales se desprende, por ejemplo, que el trabajo de carpinteros y albañiles evolucionaba a la par. Planta por planta, los muros de mampostería crecían al tiempo que el esqueleto interior de madera. Las vigas maestras se hacían trabar con la fábrica como si de un mampuesto más se tratase, lo cual dotaba al edificio de una gran robustez constructiva. Tanto es así, que parece probarse, no sólo en este caso, que es la longitud máxima que pueden alcanzar las vigas maestras (es decir, la longitud máxima aprovechable de un tronco), la que determinaba la forma en planta, y por tanto el desarrollo volumétrico de las torres ${ }^{3}$. Por ello, las dimensiones del lado corto de los torreones de planta rectangular y del lado único en los de planta cuadrangular, oscilan siempre en torno a los $11 \mathrm{~m}$ de longitud, dependiendo del grosor de los muros.

\section{APORTACIONES AL ESTUDIO DE LA FUNCIONALIDAD DEL INTERIOR DE LAS TORRES BANDERIZAS}

Esta torre de Murga era un edificio mal iluminado, mal ventilado y seguramente con serios problemas de humedad, sobre todo en la planta baja.

En cuanto al destino de los mismos, si seguimos algunos modelos propuestos recientemente, en las torres banderizas tendríamos cuatro plantas con funcionalidades concretas. La planta baja sería almacén; la primera, el salón donde se desarrollaría la mayor parte de las actividades diarias (allí estarían la cocina y el dormitorio comunitario); la segunda constituiría un espacio para la celebración de ceremonias señaladas, un lugar de representación social (GONZÁlez Cembellín, 2002), mientras que la tercera tendría una utilidad netamente defensiva, en relación con el cadalso arriba citado.

Sin embargo, ni éste ni otros de los modelos formulados hasta la fecha, parece adecuarse al caso de Murga ${ }^{4}$. Este aparente problema, más que resaltar la singularidad del to- 

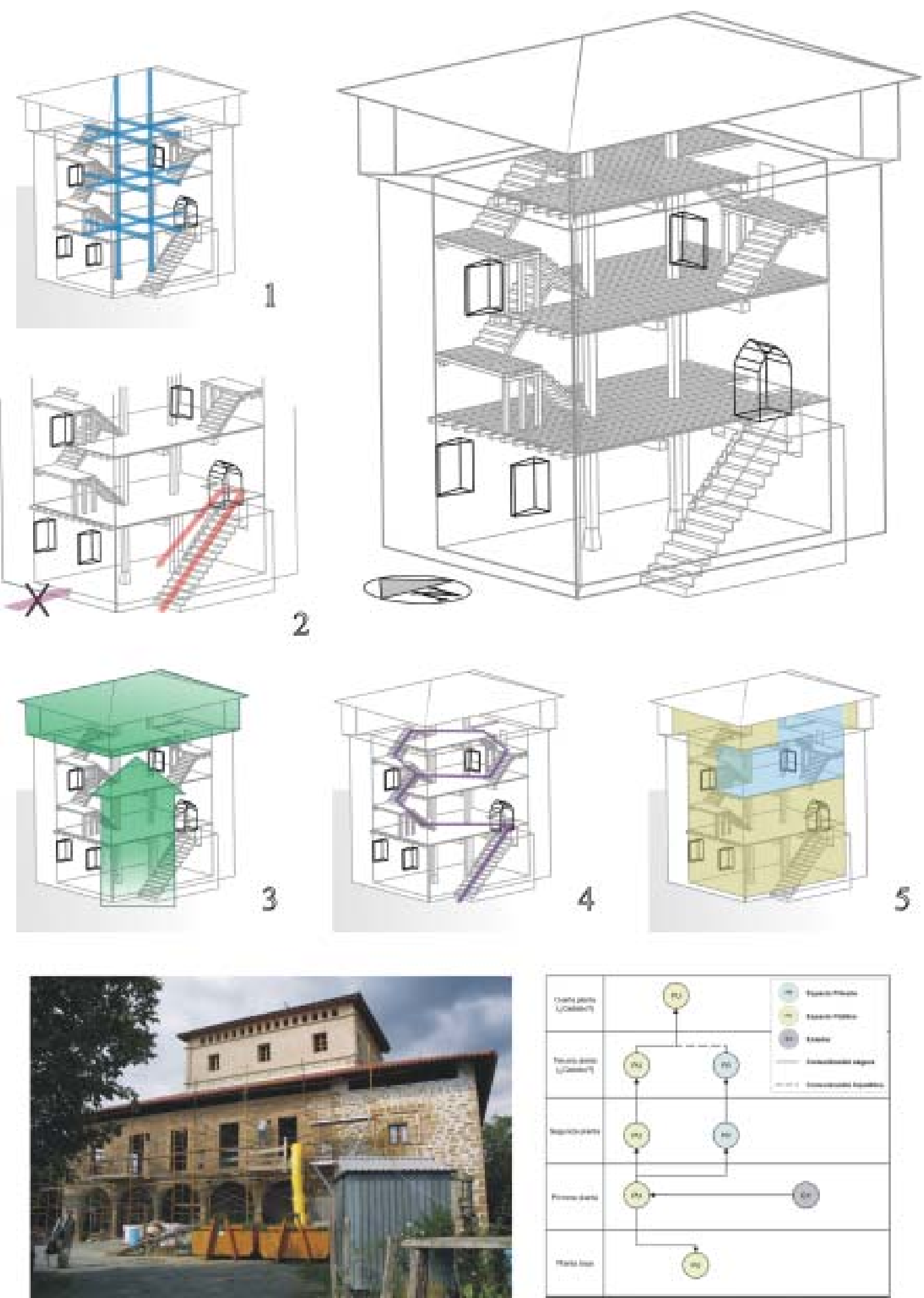

Fig. 3. En la zona superior. Reconstrucción tridimensional del interior de la torre de Murga, rodeada por varios croquis analíticos: 1. Estructura principal en madera. 2. Acceso único por la primera planta. 3. La atalaya como determinante de la circulación vertical dentro del edificio. 4. Circulación interna. 5. Espacios de uso común y espacios de uso privativo para el señor. (Modelo CAD: Amaia Mesanza). En la zona inferior. Fotografía de la torre en la actualidad y diagrama de flujos con la circulación interna y el carácter (uso común o privado) de cada uno de los espacios de la casa 
rreón, invita al cuestionamiento de nuestros conocimientos reales acerca de este tipo de entidades edilicias.

\subsection{Inexistencia de un acceso por la planta baja}

En esta torre se ha puesto de manifiesto la inexistencia de un acceso directo desde el exterior a la planta baja, ya que todos los que se observan en la actualidad fueron efectuados con posterioridad. Es decir, la única forma de llegar a este espacio era descendiendo desde la primera planta. Hecho que hace impensable su utilización como establo o cuadra y obliga a reconsiderar o definir con mayor cautela su empleo como despensa o bodega, por la dificultad que comportaría el acarreo y depósito de cargas traídas desde el exterior (Fig. 3, croquis 2). Este aparente inconveniente se convertía en una clara ventaja defensiva, pues una vez desmontado el patín de madera, alcanzar la única entrada al recinto se convertía en una misión muy complicada para el enemigo.

En realidad, la presencia de un acceso inferior en esta fase sería ilógica, de un lado porque haría redundante la existencia del que se situaba en alto y, de otro, por su vulnerabilidad, ya que una vez tomada la planta baja, la base del armazón de madera, y por tanto la propia torre, se encontraría a merced del intruso. Esta hipótesis interpretativa pone en evidencia la necesidad de realizar, en otras torres, análisis estratigráficos sistemáticos que permitan contrastar si, como sospechamos, los accesos por la planta baja son realidades constructivas ajenas a la lógica funcional de estos edificios 5 .

\subsection{Tres tramos de escalera en lugar de escalera única}

Nuestra segunda consideración parte de la constatación realizada en la torre de Murga, de la disociación de los tramos de escalera que comunicaban los diferentes pisos, condicionando así la circulación interior del edificio, y su compartimentación.

Las escaleras eran de madera, y se afianzaban sobre mechinales y ménsulas empotradas en los muros. El tramo inferior, del cual se han localizado en la excavación arqueológica dos peldaños pétreos, ascendía pegada al muro $\mathrm{E}$ y comunicaba la planta baja con la primera. (Fig. 3)

\footnotetext{
5 Hasta el momento, esto se ha podido constatar al menos en dos de las torres analizadas por nuestro Equipo: Torre de Orgaz, en Fontecha (DomíngueZ, SÁnChez, 2001) y Torre de Martiartu (GARCía, SÁNCHEZ, 2003).

${ }^{6}$ La cita documental nos ha sido proporcionada por Federico Verástegui, y se ubica cronológicamente a fines del siglo XVI.
}

El segundo tramo corría junto al muro $\mathrm{O}$, uniendo la primera y la segunda planta. En esta planta, los accesos hacia los niveles superiores de la torre se bifurcaban. Por un lado se ha registrado una escalera adosada al cierre occidental, que es continuación de la que viene del nivel inferior; al tiempo que se ha detectado la presencia de otra escalera, en la esquina sureste, que da acceso al tercer piso o al cadalso. Esta intrincada disposición tenía una clara consecuencia en la organización del espacio interior: la necesidad de cruzar las plantas para pasar de un tramo de escalera a otro, convertía la mayor parte de la superficie útil en espacio de tránsito (Fig. 3, croquis 4).

Además, en la segunda planta, encontramos una división de espacios que refleja una disociación entre lo público (la zona de paso común) y lo privado (la vivienda del senoor). Así la escalera NO, que se mantiene desde la primera planta hasta la tercera, sirve de eje vertical que comunica todos los niveles del edificio; siendo la escalera de uso común, diferente a la de la esquina SE. Esta última nace en la propia segunda planta, y da acceso al nivel superior. Debería tratarse de una escalera de uso exclusivo del señor, es decir, sería la escalera propia de su vivienda, que por lo tanto podemos deducir que se articulaba en dos plantas (Fig. 3, croquis 5 y diagrama de flujo).

En resumen, podemos establecer que la circulación vertical se da en dos planos: una escalera comunitaria, que parte de la primera planta y llega hasta el cadalso, y una escalera señorial que comunica los dos niveles en los que la vivienda del señor banderizo se articula.

\subsection{El cadalso como atalaya razón de ser de la torre banderiza}

Directamente relacionada con la anterior, realizaremos una última consideración acerca de la funcionalidad del cadalso. Entendiendo por cadalso la estructura en madera hecha en voladizo que ocupaba la parte superior de las torres, debemos reconocer que en Murga no ha quedado rastro material de su existencia, aunque sí algunas citas documentales donde se hace referencia a "la dicha torre fuerte que antiguamente tenía el remate de tabla y los corredores de afuera de lo mismo." ${ }^{6}$ Basándonos en este dato, así como en otras referencias que nos hablan de torres con cadalso (PALACIOS, 2001: 166-167; JiMÉNEZ, 1993: 78; AVELlo, 1991: 91. O en obras ya clásicas: PORTILLA, 1972: 192; YRIZAR, 1929: 31-33), y observando también el remate de ladrillo que actualmente corona el torreón, y que conserva ciertos ecos formales de aquél, damos por hecha su presencia en el edificio original (Fig. 2, ilustración fase 1).

Pues bien, una reflexión detenida sobre la configuración del interior de la torre de Murga, pone de manifiesto 
que es en éste elemento defensivo, donde reside la clave de toda su articulación espacial.

La fortaleza del torreón, la potencia de sus muros, permitía ofrecer una sólida resistencia, pero, en circunstancias de sitio, sólo la atalaya donde estaba emplazado el cadalso proporcionaba al defensor un cierto control sobre la situación. La altura a la que se encontraba no sólo proporcionaba un mayor rango de alcance a los proyectiles de las ballestas, o permitía la protección de la base del edificio, sino que sobre todo, dotaba de cobertura defensiva al entorno inmediato donde se encontraban emplazados bienes inmuebles de vital importancia para la economía del pariente mayor; en el caso de los Murga, «dos moliendas pasado el rio delante de la dicha torre» ${ }^{7}$ y algunas otras construcciones auxiliares.

Podríamos decir que, si efectivamente la construcción de una torre es la expresión de una voluntad de dominio territorial, la atalaya es el dispositivo principal que la habilita para ejercerlo ${ }^{8}$. La efectividad de ese dispositivo dependía fundamentalmente de la altura que se podía alcanzar con respecto al terreno circundante, por lo que, salvo que se pudiera aprovechar alguna colina (nunca demasiado alejada de los caminos que atravesaban el fondo de los valles), era la propia fábrica la que debía erguirse lo más alto posible. El gran desarrollo vertical de los edificios resultantes es el que obliga a articular la superficie habitable mediante varios pisos, comunicados por escaleras dispuestas de la forma anteriormente indicada. En realidad, el espacio interno de la torre no es más que el tránsito entre el suelo y el cadalso, tránsito al que se ha adaptado la vivienda común y las estancias de uso privativo por parte del pariente mayor.

\section{A MODO DE CONCLUSIÓN}

En nuestra opinión, consideraciones básicas de este tipo deben constituir el punto de partida para una revisión y mejor definición de la funcionalidad del espacio en la torre banderiza. Esta revisión debería huir de la mera descripción formal y del abuso de las clasificaciones tipológicas que tienden a crear una idea del espacio estático y fragmentado, y debería tender a una concepción más estructurada, más orgánica, donde se pudiese apreciar claramente cómo se produce el juego de relaciones espaciales, cómo se articulan las comunicaciones y cuáles son los factores que influyen en la transformación del hábitat del pariente mayor. Desde la

\footnotetext{
7 Cita documental de fines del siglo XVI, proporcionada también por Federico Verástegui.

${ }^{8}$ Desde nuestro punto de vista, funcionalmente, la torre sin cadalso en la atalaya no tiene razón de ser, lo cual constituye un argumento más para suponer su existencia en el primitivo baluarte de Murga.
}

arqueología, sólo en la medida en que seamos capaces de comprender el funcionamiento de una torre como la de Murga, estaremos en disposición de dar el siguiente paso, y de este modo, intentar definir el funcionamiento de la sociedad que la ha creado.

Presentamos resumidamente, como un ejercicio que procura ir más allá de la realidad de un único edificio, las claves para la comprensión espacial de las torres a la hora de abordar su estudio. Esta reflexión forma parte de un proyecto más amplio, que incluye el análisis de un importante elenco de torres banderizas del País Vasco, pero cuyas conclusiones trascienden ampliamente dicho entorno geográfico.

Desde nuestra experiencia, hay ciertos aspectos que resultan fundamentales para aprehender la sucesión de configuraciones internas de un edificio del tipo del que nos ocupa, que eventualmente puede ser aplicado a otro tipo de edificaciones. No nos extenderemos al tratar cada uno de esos aspectos, sino que solo definiremos sus características principales.

1. Análisis estratigráfico. Es la base imprescindible para el conocimiento detallado de cada una de las acciones que han llegado a constituir el edificio tal y como lo conocemos. El objeto de este análisis es reconstruir la secuencia en la que han ido apareciendo aquellos elementos que se conservan.

2. Reconstrucción tridimensional por fases. Este segundo «momento» del estudio puede ser dividido en tres tipos de análisis diferentes, pero que en general son realizados de manera simultánea, ya que la determinación de uno de los aspectos condiciona los demás.

2.a. Reconocimiento de los grandes volúmenes contenedores del conjunto, que normalmente coinciden con los cuerpos de fábrica principales (por ejemplo la fábrica de la torre o la del palacio adosado a la torre, etc.). Esos grandes volúmenes serán los que determinen la compartimentación interna y la configuración de los espacios que de ella se deriva.

2.b. Delimitación de los espacios internos. Cada uno de los volúmenes definidos en el apartado anterior puede estar compartimentado, segmentación que en nuestro caso es difícil de visualizar al tratarse de estructuras lígneas desaparecidas, que no dejaron restos en los muros de piedra.

2.c. Identificación de los accesos. Este paso está íntimamente ligado al anterior, siendo en gran medida su consecuencia ineludible. El análisis de la distribución de los vanos, accesos y escaleras es fundamental, permitiéndonos llegar a conocer la distribución interna del cuerpo principal 
así como sus relaciones con los cuerpos adyacentes y el exterior. Estos accesos marcan la relación física entre espacios, pero lo que es más importante, las relaciones entre las personas que utilizaban la torre. Así, podremos determinar grados de permeabilidad, relaciones de preeminencia, gradientes de visualización, etc., gracias a los cuales llegaremos a comprender una cierta idea de las relaciones personales para cada momento analizado.

3. Estudio de la circulación o circuitos. El conocimiento tridimensional del edificio en sus diferentes fases, por el que se reconoce para cada momento la red de vanos y accesos (tanto si comunican la torre con el exterior como si comunican espacios internos del edificio), permite establecer unas redes de circulación sin las cuales no es posible la comprensión integral de la torre. Los cambios en la circulación que supone la abertura de nuevas puertas, por ejemplo, puede indicarnos modificaciones importantes en la concepción del propio edificio y su funcionalidad, así como el status de sus moradores.

Por último, queremos resaltar que la idea directriz de nuestra propuesta de trabajo es que este tipo de estudios debe ser realizado en función de la articulación del espacio como un sistema. Las numerosas interrelaciones de los aspectos reseñados nos muestran que sería poco viable analizar unos aspectos sin tener en cuenta los demás, ya que cualquier modificación en uno de ellos va a tener repercusión en los demás. Por lo tanto debemos estudiar cada conjunto edilicio en términos de sistema, analizando las interacciones multidireccionales que se operan entre cada uno de los aspectos, teniendo siempre como base las relaciones que se establecen sincrónicamente, para luego estudiar las alteraciones introducidas en el sistema, representando la diacronía, es decir, la secuencia histórico-constructiva.

\section{Bibliografía}

Avello J. L., 1991, Las torres señoriales de la Baja Edad Media asturiana, León.

Díaz de DuRANa J. R., 1998, Historia y presente del tratamiento historiográfico sobre la Lucha de Bandos en el País Vasco. Balance y perspectivas al inicio de una nueva investigación, en La lucha de bandos en el Pais Vasco, de los parientes mayores a la hidalguía universal: Guipuzcoa, de los bandos a la provincia (siglos XIV a XVI), Bilbao.

Domínguez I. C., SÁNChez ZufiaurRe L., 2001, De la teoría a la práctica: Aplicación del método de lectura estratigráfica a la Torre de Orgaz, en Fontecha (Álava), Actas del V Congreso de Arqueología Medieval Española, Valladolid, pp. 213-220.

García Gómez I, SÁnChez PinTo I, 2003, Documentación estratigráfica y análisis histórico de la torre de Martiartu (Erandio, Bizkaia), $A r-$ keoikuska 02, pp. 467-469.

GonZÁlez Cembellín J. M., 2002, Las casas-torre en Bizkaia de los siglos XV y XVI. El caso portugalujo, en Díaz De Durana J. R., RegUeRA, I. (Eds.), Lope García de Salazar: banderizo y cronista. Actas de las II Jornadas de Estudios Históricos Noble villa de Portugalete, Portugalete, pp. 65-93.

JiméNEZ J. M., 1993, Torres y castillos de la Cantabria medieval, Santander.

LEMA Pueyo J. A., et al, 2000, Los señores de la guerra y de la tierra: nuevos textos para el estudio de los Parientes Mayores guipuzcoanos (1265-1548), San Sebastián.

Palacios V.,2001, El patrimonio artístico del Valle de Ayala: Torres, Palacios y puentes, en García Fernández E. (Coord.), La tierra de Ayala. Actas de las Jornadas de Estudios Históricos en conmemoración del 600 Aniversario de la construcción de la Torre de Quejana, Vitoria, pp. 163-189.

Portilla Vitoria M. J., 1972, Torres y casas fuertes en Álava, Vitoria. YRIZAR J. DE, 1929, Las casas vascas, Bilbao. 